\title{
Cyclic voltammetric determination of capsaicin by using electrochemically deposited tin and reduced graphene oxide on screen-printed carbon electrodes
}

\author{
Wasukamol Numphud, Orapin Chienthavorn, Wilai Siriwatcharapiboon* \\ Department of Chemistry and the Center of Excellence for Innovation in Chemistry, Faculty of Science, \\ Kasetsart University, Bangkok 10903 Thailand
}

*Corresponding author, e-mail: fsciwls@ku.ac.th

Received 28 May 2020

Accepted 19 Aug 2020

\begin{abstract}
Tin and reduced graphene oxide ( $\mathrm{Sn} / \mathrm{rGO}$ ) was prepared by an electrochemical deposition and modified on a screen-printed carbon electrode (SPCE) in order to improve the electrode selectivity and sensitivity for determination of capsaicin in real samples. The modified electrode was characterized for its surface morphology by scanning electron microscopy (SEM). An elemental analysis of prepared catalysts was confirmed by energy dispersive spectroscopy (EDS) and x-ray photoelectron spectroscopy (XPS). The experimental conditions influencing determination of capsaicin were optimized. The experiment was carried out in a sodium acetate buffer solution $\mathrm{pH} 3.0$ at a scan rate of $50 \mathrm{mV} / \mathrm{s}$. The $\mathrm{Sn} / \mathrm{rGO} / \mathrm{SPCE}$ showed a linear working range of $0.2-22 \mu \mathrm{M}$ of capsaicin concentrations. The limit of detection and limit of quantification were $0.005 \mu \mathrm{M}(\mathrm{S} / \mathrm{N}=3)$ and $0.02 \mu \mathrm{M}(\mathrm{S} / \mathrm{N}=10)$, respectively. The prepared electrode was successfully applied to determine capsaicin in real chili samples and chili sauces.
\end{abstract}

KEYWORDS: capsaicin detection, modified electrode, electrocatalyst, electrochemical sensor

\section{INTRODUCTION}

Chili is one of the most popular ingredients in food owing to its unique characteristics for desirable taste and improving appetite [1]. The spiciness and pungency of chili pepper is attributed to a compound group known as "capsaicinoids", which are found in members of the capsicum family [2, 3]. Capsaicin $\left(\mathrm{C}_{18} \mathrm{H}_{27} \mathrm{NO}_{3}\right)$ is a main compound in the capsaicinoid group that includes dihydrocapsaicin, homocapsaicin and homodihydrocapsaicin [4-6]. Capsaicin has been widely used in pharmaceutical and medical applications due to its remarkable properties, such as boosting metabolism to increase amount of heat in body and burn more calories [7], reducing blood pressure [8], helping dilation of blood vessel [9], and improving blood circulation [10]. Capsaicin also has antioxidative [11], antibacterial [12] and anticarcinogenic [13] properties.

Due to the importance of capsaicin in culinary and biomedical uses, a simple, rapid and accurate determination of capsaicin is necessary, particularly in food and pharmaceutical industries. Several analytical techniques for determination of capsaicin, such as spectrophotometry, gas chromatography (GC) [14], high performance liquid chromatography (HPLC) [6], gas chromatographymass spectrometry (GC-MS) [15], and liquid chromatography-mass spectrometry (LC-MS) [16], have been reported. However, these methods require the use of expensive instruments with complicated procedure and long response time. Alternate approach for capsaicin determination could be done via electrochemistry due to its advantages, such as high efficiency, good reproducibility, greater linear response range, and easy operation. Among electrochemical techniques, cyclic voltammetry is the most widely used to study the electrochemical behavior of electroactive species by using biosensor $[17,18]$ or non-biosensor [19].

Electrochemical determination of capsaicin was firstly reported by Kachoosangi et al [20] by using adsorptive stripping voltammetry at a carbon nanotube (CNT) based electrochemical sensors. Other approaches were reported on different types of electrodes for detection of capsaicin, such as boron-doped diamond electrode [21], glassy carbon electrode [22], carbon nanotube (CNT) [19], graphene oxide (GO) [23], reduced graphene oxide (rGO) [24], precious metal [25, 26], non-precious metals [23], carbon paste electrode modified with amino-functionalized mesoporous silica [27], and 
screen printed single-walled CNT [19]. Furthermore, several metallic nanoparticles modified electrode surface and their enhanced sensitivity and selectivity were also investigated, for example, $\mathrm{Ag} / \mathrm{Ag}_{2} \mathrm{O}$ functionalized reduced graphene oxide (rGO) on screen-printed electrode (SPE) [25] and ruthenium nanoparticles decorated CNTs [28]. The precious metals always show high catalytic activity; however, the obtained electrodes have a high cost of production. The complicated and expensive electrode fabrication process may restrict the improving of capsaicin sensor in the future. Hence, a disposable, cheap, sensitive, and selective electroanalytical sensor for determination of capsaicin is necessary.

The screen-printed carbon electrode (SPCE) is a low cost sensor. It can be modified and tailored to appropriately serve the demand of the end user and offer high potential for commercial applications. As the sensitivity and selectivity of the SPCE towards the target molecules are imperative, the reduced graphene oxide ( $\mathrm{rGO}$ ), a derivative of graphene, has attracted tremendous attentions due to its properties, such as high surface area and excellent electrochemical properties. However, the limited conductivity of the rGO and its poor dispersibility are still challenging factors for the rGO modified electrode. In order to solve these problems, rGO has been functionalized with precious metals $[25,26]$ and non-precious metals [22]. Tin (Sn) is a pblock metal that has been widely used for promoting adsorption of several molecules on an electrode surface $[29,30]$. Tin can supply highly oxophilic sites where one oxygen atom in the target molecule can be adsorbed on the electrode surface. It is possible for tin to form a key part of active site, either purely as a coordination site or indeed as part of the redox system. Furthermore, tin has been used as a catalyst due to its high selectivity and performance to facilitate electron transfer between electroactive species and electrode surface [31,32].

Based on the facts mentioned above, we aimed to develop disposable electrochemical sensors by using non precious metal, rGO and SPCE. Therefore, $\mathrm{Sn}$ and rGO were employed to enhance electrocatalytic activity of SPCE for determination of capsaicin in our present work. The Sn/rGO/SPCE was prepared by using electrochemical deposition. Investigation of capsaicin adsorption on the newly modified electrode was then achieved by cyclic voltammetry. The electrode was subsequently used for determination of capsaicin in Bird's eye chili, Karen chili and chilli sauces.

\section{MATERIALS AND METHODS}

\section{Reagents}

Capsaicin $\left(\mathrm{C}_{18} \mathrm{H}_{27} \mathrm{NO}_{3}\right)$ with $\geqslant 99 \%$ purity, tin (II) chloride dihydrate $\left(\mathrm{SnCl}_{2} \cdot 2 \mathrm{H}_{2} \mathrm{O}\right)$ with $\geqslant 99.995 \%$ purity and graphene oxide $(2 \mathrm{mg} / \mathrm{ml}$ dispersion in $\mathrm{H}_{2} \mathrm{O}$ ) were obtained from Sigma-Aldrich (St. Louis, USA). Standard tested interfering substances, such as D-(+)-glucose, D-(-)-fructose with $\geqslant 99 \%$ purity, zinc (II) chloride anhydrous with $\geqslant 97 \% \mathrm{pu}-$ rity, copper (II) chloride were also obtained from Sigma-Aldrich (St. Louis, USA). Potassium chloride and sodium hydroxide anhydrous pellets were purchased from Carlo Erba Reagents (Barcelona, Spain). The electrolyte solution was a mixture of glacial acetic acid and anhydrous sodium acetate obtained from Carlo Erba reagents (Barcelona, Spain) and Sigma-Aldrich (St. Louis, USA), respectively. Carbon paste and $\mathrm{Ag} / \mathrm{AgCl}$ paste were purchased from SunChemical (Torfaen, UK).

\section{Preparation of tin and reduced graphene oxide screen-printed carbon electrode ( $\mathrm{Sn} / \mathrm{rGO} / \mathrm{SPCE})$}

A screen-printed technique was applied for making electrodes on a chip for electrochemical measurement. Carbon paste was screen-printed onto a $10 \mathrm{~mm} \times 25 \mathrm{~mm}$ of polyvinyl chloride (PVC) sheet (Paper Thai Co., Ltd.) and dried at $60^{\circ} \mathrm{C}$ for $30 \mathrm{~min}$ to form a working electrode area of $3 \mathrm{~mm}$ diameter and a counter electrode. After that, $\mathrm{Ag} / \mathrm{AgCl}$ paste was applied onto the pre-screened PVC to form a reference electrode and dried at $60^{\circ} \mathrm{C}$ overnight. An insulating ink was finally screen-printed onto three electrodes to exclude the detection area. The printed carbon electrode was dried at room temperature and kept in a desiccator.

A mixture of $0.5 \mathrm{ml} \mathrm{GO}, 103 \mu \mathrm{l}$ of $0.05 \mathrm{mM}$ tin (II) chloride solution and $1.4 \mathrm{ml}$ deionized water was sonicated for $1 \mathrm{~h}$ to prepare tin and graphene oxide (Sn/GO) catalyst ink. A $6 \mu 1 \mathrm{Sn} / \mathrm{GO}$ catalyst ink was drop-casted on the working electrode of the bare SPCE and dried at room temperature. A $100 \mu \mathrm{l}$ sodium acetate buffer solution was dropped to cover all modified SPCE surface, and the electrode was subjected to chronoamperometry with an applied potential of $-0.44 \mathrm{~V}$ for $5 \mathrm{~min}$ to obtain a Sn/rGO/SPCE. To obtain optimum potential and time for Sn electrodeposition, the potentials and time were varied, as shown in Fig. S1 in supplementary data. The potential of $-0.44 \mathrm{~V}$ and the deposition time for $300 \mathrm{~s}$ showed the maximum peak current. The potential of electrode was controlled by the Autolab PGstat20 potentiostat (Metrohm-Autolab, 
Netherlands).

\section{Electrochemical measurement}

A $0.00764 \mathrm{~g}$ of capsaicin powder was dissolved in $250 \mathrm{ml}$ sodium acetate buffer solution $(\mathrm{pH} 3.0)$ to obtain $100 \mu \mathrm{M}$ capsaicin stock solution. The stock solution was then diluted with sodium acetate buffer solution ( $\mathrm{pH}$ 3.0) to obtain capsaicin standard solutions in a concentration range of $0.2-22 \mu \mathrm{M}$. For the the study of capsaicin determination, the cyclic voltammetric (CV) measurement was conducted over the range from 0 to $1.0 \mathrm{~V}$ at a scan rate of $50 \mathrm{mV} / \mathrm{s}$ in $100 \mu \mathrm{l}$ of $0.01 \mathrm{M}$ sodium acetate buffer solution ( $\mathrm{pH}$ 3.0) to check the performance of the electrode. The sodium acetate buffer solution was removed. A $100 \mu \mathrm{l}$ of the capsaicin standard solution was then dropped on the electrode surface for the determination of capsaicin at a scan rate of $50 \mathrm{mV} / \mathrm{s}$.

\section{Preparation of real samples}

The as-prepared Sn/rGO/SPEC electrode was used to test the electrochemical determination of capsaicin in real samples. Bird's eye chilli, Karen chili and chili sauces were obtained from a local market in Bangkok. The extraction of capsaicin from the real samples was carried out following procedure of Ya et al [27]. A $0.2 \mathrm{~g}$ ground sample was mixed with $25 \mathrm{ml}$ ethanol, then sonicated for $1 \mathrm{~h}$, centrifuged at $12000 \mathrm{rpm}$ for $20 \mathrm{~min}$, and filtered. The supernatant was diluted with sodium acetate buffer solution (pH 3.0) to obtain $50 \mathrm{ml}$ stock solution of chili samples.

A standard addition method was adopted to measure capsaicin in real samples. A $100 \mu \mathrm{M}$ of capsaicin standard solution was spiked into the stock solution of chili samples to give the concentration of $5,10,15$, and $20 \mu \mathrm{M}$ in $0.01 \mathrm{M}$ sodium acetate buffer solution ( $\mathrm{pH}$ 3.0). The CV measurements of spiked and non-spiked samples were carried out under the optimum condition at $50 \mathrm{mV} / \mathrm{s}$. The obtained results were calculated for the percentage recovery $(\% \mathrm{R})$ as follows:

$$
\% R=\frac{C_{S}-C_{U}}{C_{A}} \times 100
$$

where $C_{S}=$ concentration of capsaicin in spiked sample, $C_{U}=$ concentration of capsaicin in unspiked sample and $C_{A}=$ concentration of capsaicin added in the unspiked sample. Calibration curves in the range of $0.2-22 \mu \mathrm{M}$ were used for the calculation of capsaicin concentrations in the chili samples.
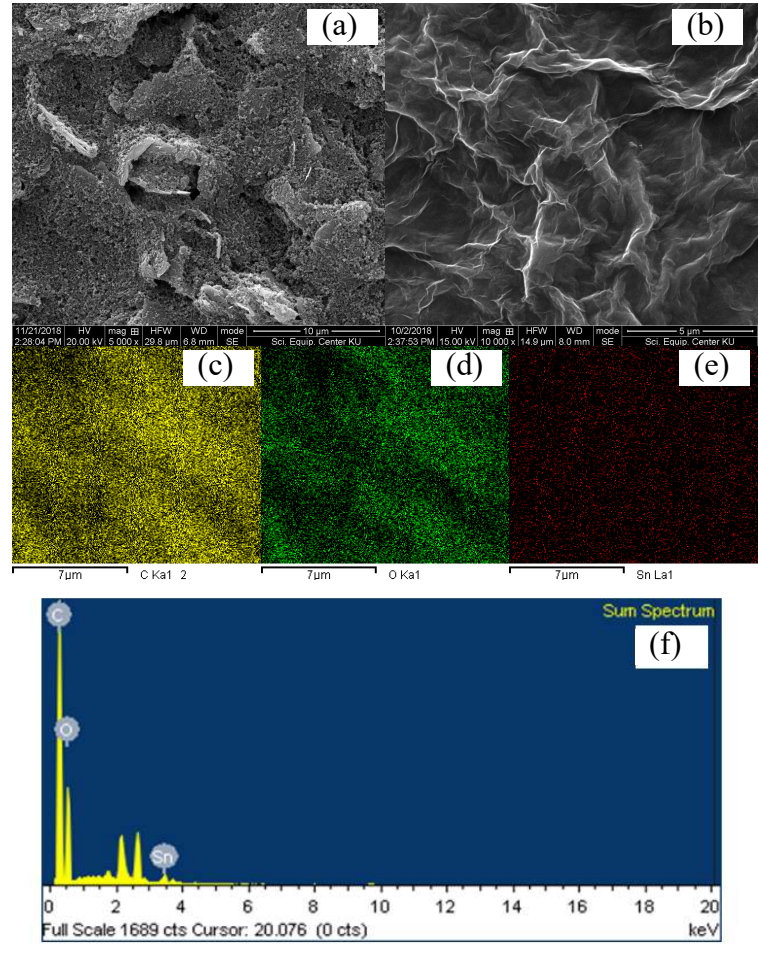

Fig. 1 SEM images of (a) bare SPCE and (b) Sn/rGO/SPCE with elemental mapping of (c) carbon, (d) oxygen, (e) tin, and (f) EDS spectra of Sn/rGO/SPCE.

The experiment was repeated three times for each concentration and the relative standard deviation (RSD) was calculated.

\section{RESULTS AND DISCUSSION}

\section{Characterization of $\mathrm{Sn} / \mathrm{rGO} / \mathrm{SPCE}$}

SEM images of bare SPCE and Sn/rGO/SPCE are shown in Fig. 1a and Fig. 1b, respectively. A distinct difference in the surface morphology was observed between the bare SPCE and the Sn/rGO/SPCE. The bare SPCE showed roughness of carbon sheet, while the $\mathrm{Sn} / \mathrm{rGO} / \mathrm{SPCE}$ showed a cloudy and wrinkled texture of rGO sheets that fully covered on the SPCE surface. The spherical-shaped particles of Sn were however not observed on the rGO sheets because they were smaller than the resolution limitation of the instrument and possibly under rGO sheets. Fig. 1(c,d,e) show a composition of $\mathrm{Sn} / \mathrm{rGO}$ by elemental mapping of carbon, oxygen and tin, respectively. The EDS analysis of Sn/rGO/SPCE gave the spectra of carbon and oxygen at 0.27 and $0.52 \mathrm{keV}$ [33] (Fig. 1f), confirming the elemental composition of rGO. Although, no Sn particle presented in the SEM image, a signal of Sn at $3.44 \mathrm{keV}$ 

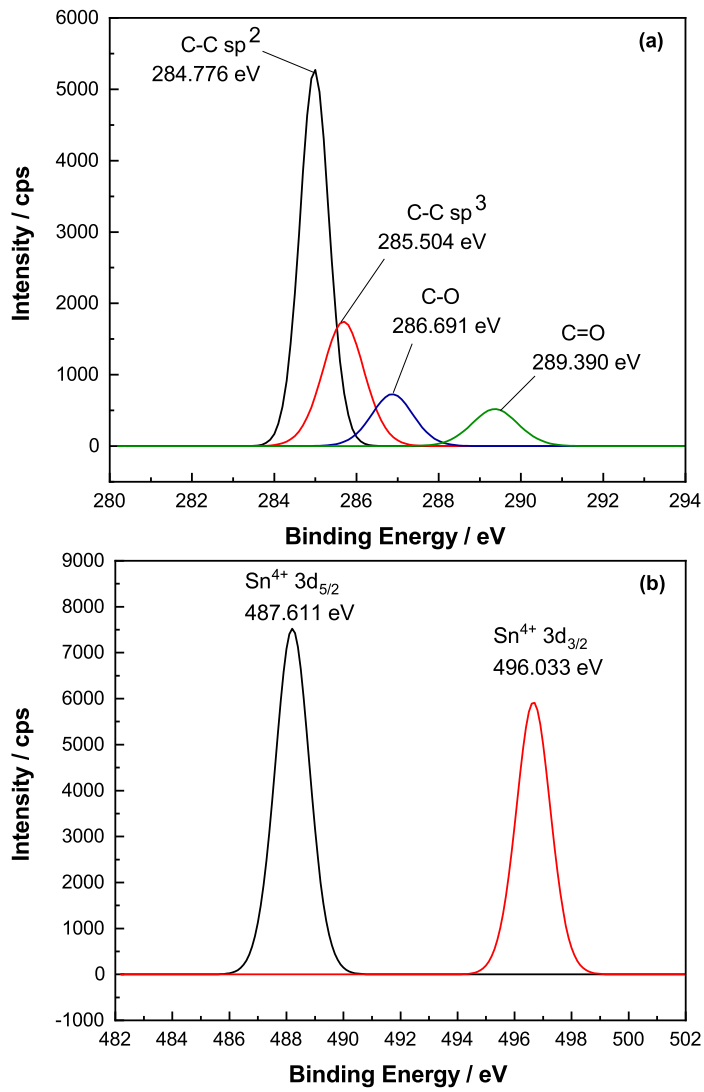

Fig. 2 High resolution XPS spectra of (a) C 1s and (b) $\mathrm{Sn} 3 \mathrm{~d}$ of $\mathrm{Sn} / \mathrm{rGO} / \mathrm{SPCE}$.

was observed. This could be a confirmation for the presence of $\mathrm{Sn}$ in the modified $\mathrm{Sn} / \mathrm{rGO} / \mathrm{SPCE}$ electrode [34].

Surface composition and electronic valence states of the $\mathrm{Sn} / \mathrm{rGO} / \mathrm{SPCE}$ were confirmed by using XPS technique. Fig. 2a shows the $\mathrm{C} 1 \mathrm{~s}$ spectrum at $284.7 \mathrm{eV}$, which indicates the formation of $\mathrm{C}-\mathrm{C}$ $\mathrm{sp}^{2}$. In addition, the spectra of $\mathrm{C} 1 \mathrm{~s}$ were deconvoluted into three peaks of $\mathrm{C}-\mathrm{C} \mathrm{sp}{ }^{3}$ at $285.5 \mathrm{eV}$, $\mathrm{C}-\mathrm{O}$ at $286.7 \mathrm{eV}$ and $\mathrm{C}=\mathrm{O}$ at $289.4 \mathrm{eV}[34,35]$. The $\mathrm{Sn} 3 \mathrm{~d}$ spectrum in Fig. 2b shows two de-convoluted peaks of which the binding energy at $487.6 \mathrm{eV}$ and $496.0 \mathrm{eV}$, corresponding to the $\mathrm{Sn} 3 \mathrm{~d}_{5 / 2}$ and $\mathrm{Sn} 3 \mathrm{~d}_{3 / 2}$ of $\mathrm{Sn}^{2+/ 4+}$ [36]. No doublet due to metallic $\mathrm{Sn}$ (493.2 and $484.8 \mathrm{eV}$ ) was observed, suggesting that $\mathrm{Sn}$ is easily oxidized at room temperature $[37,38]$.

\section{Electrochemical behavior of Sn/rGO/SPCE on capsaicin determination}

To study the electrochemical performance of the prepared SPCE, CV measurement was used to determine capsaicin in sodium acetate buffer solution.

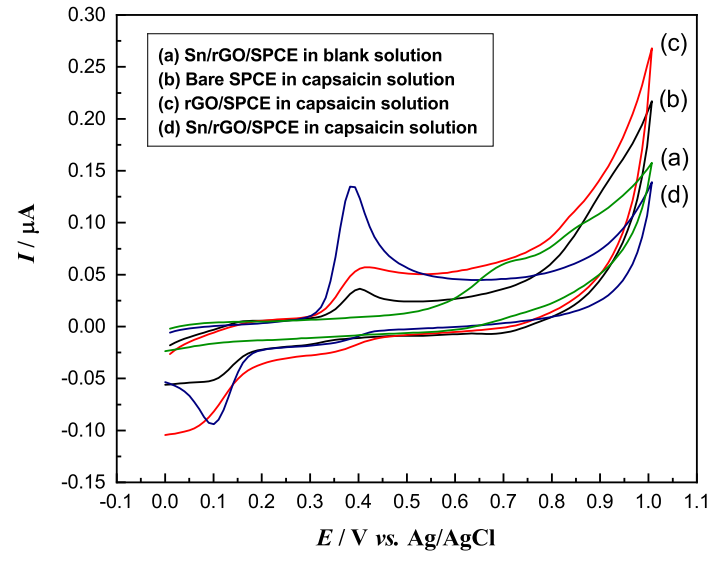

Fig. 3 Cyclic voltammograms in $0.01 \mathrm{M}$ sodium acetate buffer solution (pH 3.0) of (a) $\mathrm{Sn} / \mathrm{rGO} / \mathrm{SPCE}$, in $2 \mu \mathrm{M}$ capsaicin and sodium acetate buffer solution ( $\mathrm{pH}$ 3.0) of (b) bare SPCE, (c) rGO/SPCE and (d) Sn/SPCE/rGO at $50 \mathrm{mV} / \mathrm{s}$.

Fig. 3 shows the cyclic voltammograms of the $\mathrm{Sn} / \mathrm{r}$ GO/SPCE in a blank 0.01 M sodium acetate buffer solution, the bare SPCE, the $\mathrm{rGO} / \mathrm{SPCE}$ and the $\mathrm{Sn} / \mathrm{rGO} / \mathrm{SPCE}$ in $2 \mu \mathrm{M}$ capsaicin solution at the scan rate of $50 \mathrm{mV} / \mathrm{s}$. The cyclic voltammogram signal of the $\mathrm{Sn} / \mathrm{rGO} / \mathrm{SPCE}$ obtained in $0.01 \mathrm{M}$ sodium acetate buffer solution ( $\mathrm{pH}$ 3.0) showed a peak at $E_{\mathrm{pa}}=+0.70 \mathrm{~V}$, which was attributed to tin oxide formation. Compared with the $\mathrm{Sn} / \mathrm{rGO} / \mathrm{SPCE}$ in blank solution, the SPCE, the rGO/SPCE and the $\mathrm{Sn} / \mathrm{rGO} / \mathrm{SPCE}$ in $2 \mu \mathrm{M}$ capsaicin solution showed the oxidation peak at $E_{\mathrm{pa}}=+0.40 \mathrm{~V}$ on the forward scan, which was capsaicin oxidation. However, the $E_{\mathrm{pa}}=+0.70 \mathrm{~V}$ was discernible in a presence of capsaicin since the adsorption of capsaicin on the catalyst surface suppressed the presence of this anodic peak. The backward scan demonstrated the peak at $E_{\mathrm{pc}}=+0.10 \mathrm{~V}$, which was the reduction of $O$-benzoquinone. The position of the cathodic peak was however far from that of anodic peak, suggesting a sluggish reduction process. An oxidation peak and another pair of redox peaks related to the capsaicin reactions at the surface of the bare SPCE and the $\mathrm{Sn} / \mathrm{rGO} / \mathrm{SPCE}$ are shown, which are in accordance with the mechanism in Fig. 5. This mechanism was proposed in the previous report [18]. The electrochemical oxidation of capsaicinoids is an irreversible step. It involves the oxidation of phenolic hydroxyl group and the hydrolysis of the methoxy group, resulting in the production of an $O$-benzoquinone unit $[20,21]$. The oxidative peak at $+0.40 \mathrm{~V}$ showed the highest sensitivity compared 

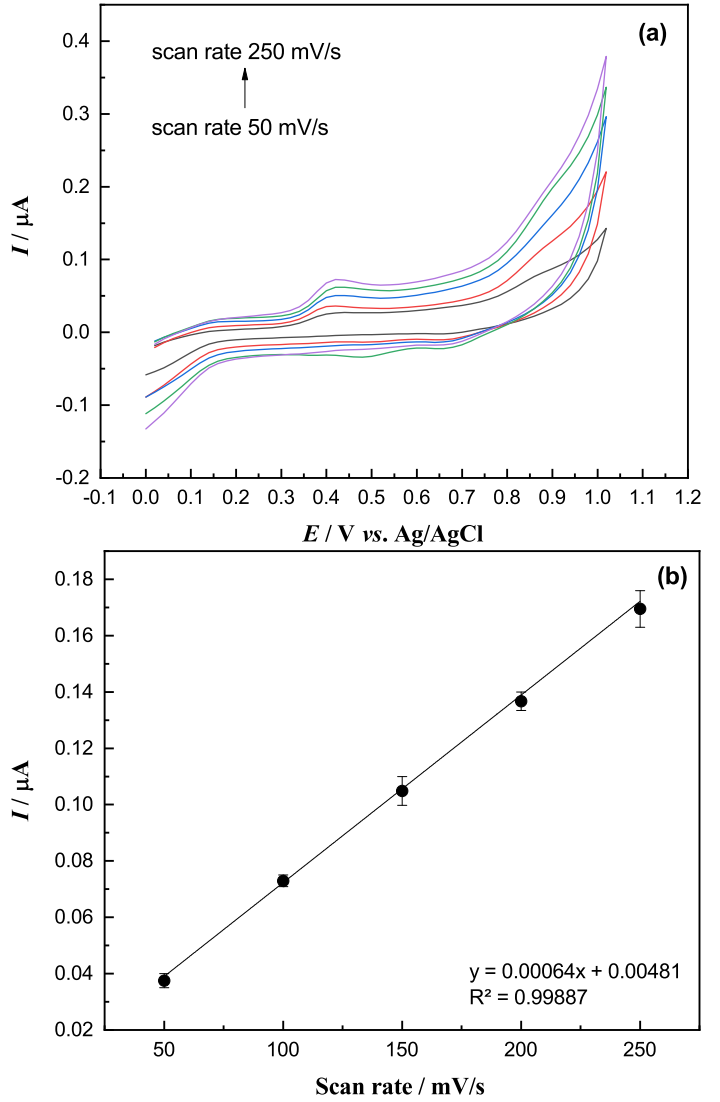

Fig. 4 (a) Cyclic voltammograms of $\mathrm{Sn} / \mathrm{rGO} / \mathrm{SPCE}$ and (b) linear plot for anodic peak of Sn/rGO/SPCE in $2 \mu \mathrm{M}$ capsaicin and sodium acetate buffer solution ( $\mathrm{pH}$ 3.0) at difference scan rates from 50 to $250 \mathrm{mV} / \mathrm{s}$.

with the cathodic peak. It was then selected to quantify capsaicin in chilli samples. The anodic peak current of capsaicin on the $\mathrm{Sn} / \mathrm{rGO} / \mathrm{SPCE}$ was significantly higher than those on the $\mathrm{rGO} / \mathrm{SPCE}$ and the bare SPCE, indicating that the presence of Sn can enhance the sensitivity of the electrode. The catalytic performance of the $\mathrm{Sn} / \mathrm{rGO} / \mathrm{SPCE}$ was possibly attributed to the uniform distribution of $\mathrm{Sn}$ over rGO sheet, which resulted in the active site on the prepared SPCE. Furthermore, the presence of Sn on the SPCE surface increased the adsorption of capsaicin and facilitated the electron transfer in capsaicin oxidation. Therefore, the $\mathrm{Sn} / \mathrm{rGO}$ modified SPCE electrochemical sensor could be potentially used for the detection of capsaicin.

\section{Effect of scan rate on capsaicin determination}

Electrochemical behaviors of capsaicin were investigated at different scan rates, as shown in Fig. 4. The cyclic voltammograms of the Sn/rGO/SPCE for

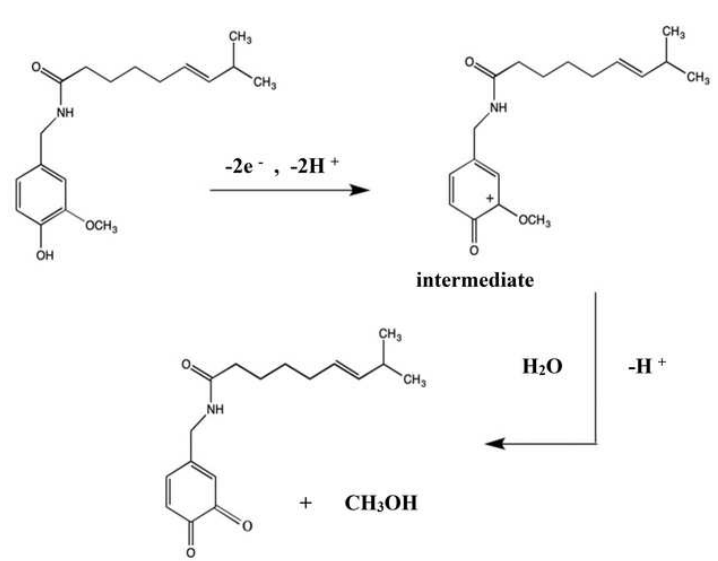

Fig. 5 Mechanism of the electrochemical oxidation of capsaicin [20].

$2 \mu \mathrm{M}$ capsaicin in sodium acetate buffer solution $(\mathrm{pH}$ 3.0) were determined at different scan rates, ranging from 50 to $250 \mathrm{mV} / \mathrm{s}$. The effect of scan rate on peak potential of capsaicin oxidation was investigated. The anodic peak potential positively shifted as the scan rate increased (Fig. 4a). The anodic currents of capsaicin improved with increasing scan rate. A good linear relationship between the peak current and the scan rate was obtained with a regression equation of $I_{\mathrm{pa}}=0.00064 v+0.00481$ $\left(R^{2}=0.998\right)$ (Fig. 4b). It was apparent that the anodic peak currents were linear with the scan rates, indicating that the electrochemical oxidation of capsaicin on the $\mathrm{Sn} / \mathrm{rGO} / \mathrm{SPCE}$ was an adsorption controlled process.

\section{Effect of $\mathrm{pH}$ on capsaicin determination}

A study of $\mathrm{pH}$ affecting the capsaicin oxidation in the $\mathrm{pH}$ range of 1.0-6.0 in sodium acetate buffer solution demonstrated that the anodic peak currents of capsaicin increased with rising $\mathrm{pH}$ and reached the maximum at $\mathrm{pH}$ 3.0, and dropped afterwards, as shown in Fig. S2. The pH of electrolyte solution clearly affected anodic currents due to hydrolysis of 2-methoxy group in capsaicin molecule, as shown in the mechanism in Fig. 5 [20]. At $\mathrm{pH}>3.0$ the $O$-methoxyphenol moiety of capsaicin was partially deprotonated, resulting in inefficient accumulation of capsaicin on the Sn/rGO/SPCE [20]. At the $\mathrm{pH}$ lower than 3.0, the peak currents dramatically decreased, since high amounts of protons in the acidic solution adsorbed on the tin oxide, resulting in less amount of capsaicin on the surface of the $\mathrm{Sn} / \mathrm{rGO} / \mathrm{SPCE}$. Thus, the sodium acetate buffer so- 

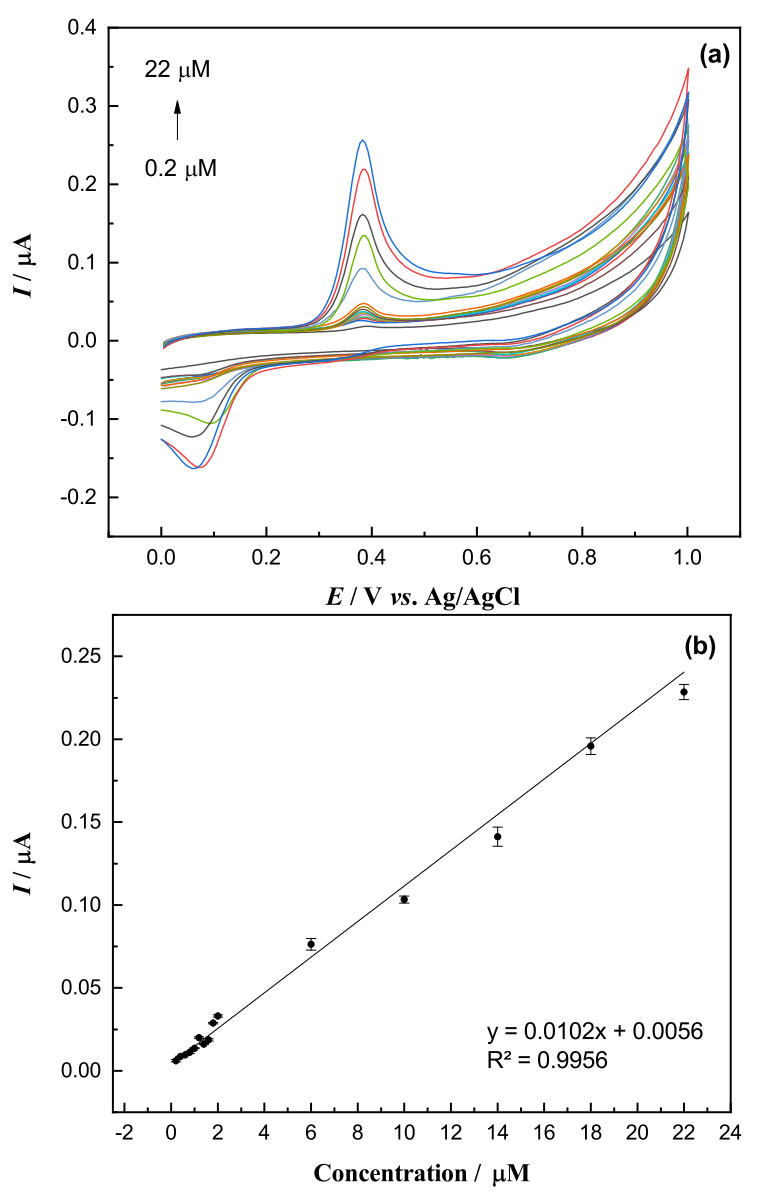

Fig. 6 (a) Cyclic voltammograms and (b) linear plots between concentration of capsaicin and anodic peak current in $0.2-22 \mu \mathrm{M}$ capsaicin and sodium acetate buffer solution (pH 3.0) at $50 \mathrm{mV} / \mathrm{s}$.

Table 1 Comparison of efficiency of Sn/rGO/SPCE with literature of modified electrodes for capsaicin determination.

\begin{tabular}{lccc}
\hline Modified electrode & $\begin{array}{c}\text { LOD } \\
(\mu \mathrm{M})\end{array}$ & $\begin{array}{c}\text { Linear range } \\
(\mu \mathrm{M})\end{array}$ & Ref. \\
\hline PDDA-rGO/Pd modified GCE & 0.10 & $0.32-64$ & {$[29]$} \\
MCFs modified CPE & 0.08 & $0.76-11.65$ & {$[39]$} \\
$\mathrm{Ag} / \mathrm{Ag}_{2}$ O-PSS-rGO modified SPE & 0.40 & $1.0-60$ & {$[28]$} \\
MWCNT modified BPPGE & 0.31 & $0.5-60$ & {$[20]$} \\
Sn/rGO modified SPCE (this work) & 0.005 & $0.2-22$ & \\
\hline
\end{tabular}

lution $\mathrm{pH} 3.0$ was chosen as the optimized value for capsaicin detection.

\section{Electrochemical determination of capsaicin}

A relationship between the anodic peak current and concentration of capsaicin was investigated by using cyclic voltammetry under the optimal conditions. Fig. 6a shows cyclic voltammograms obtained from different concentrations of standard capsaicin solution ranging from $0.2 \mu \mathrm{M}$ to $22 \mu \mathrm{M}$. The anodic and cathodic peak currents were presented at $+0.40 \mathrm{~V}$ and $+0.10 \mathrm{~V}$ on the forward and backward scans, respectively. At a potential of $+0.40 \mathrm{~V}$, the anodic peak currents increased as a function of capsaicin concentrations. Fig. 6b shows a linear calibration plot of the capsaicin concentrations and the anodic peak currents measured by using the $\mathrm{Sn} / \mathrm{rGO} / \mathrm{SPCE}$, giving a regression equation of $I_{\mathrm{pa}}(\mu \mathrm{A})=0.0102$ $C(\mu \mathrm{M})+0.0056$ with $R^{2}=0.9956$. The limit of detection (LOD) and the limit of quantification (LOQ) were estimated at $0.005 \mu \mathrm{M}(\mathrm{S} / \mathrm{N}=3)$ and $0.02 \mu \mathrm{M}(\mathrm{S} / \mathrm{N}=10)$, respectively. It was noticed that the precision represented by the error bar of each standard solution was remarkably good, ranging between $1.29 \%$ RSD and $4.50 \%$ RSD of the lowest concentration in the range. A comparison of the $\mathrm{Sn} / \mathrm{rGO} / \mathrm{SPCE}$ efficiency with those of other modified electrodes was given in Table 1 . The $\mathrm{Sn} / \mathrm{r}$ GO/SPCE showed lower LOD and a linear range comparing to the previously purposed electrodes. These results were due to the oxophilicity of tin and its favourable interaction with capsaicin on the surface of Sn/rGO/SPCE. Therefore, tin increased the adsorption of capsaicin on the electrode surface along with its catalytic activity. Furthermore, the dispersion of tin on the rGO can increase surface area and active sites of the catalyst, proving an excellent quantitative performance for sensitive and rapid capsaicin determination by using the proposed $\mathrm{Sn} / \mathrm{rGO} / \mathrm{SPCE}$. However, the mechanism of the capsaicin oxidation was similar to the mechanism in Fig. 5 proposed in the previous report [20].

\section{Electrochemical determination of interference}

Chili and its food products usually contain additives and chemical substances, such as $\mathrm{K}^{+}, \mathrm{Zn}^{2+}, \mathrm{Na}^{+}$, $\mathrm{Cu}^{2+}$, glucose and fructose [32]. These compounds can possibly disturb the electrooxidation of capsaicin. Therefore, the selectivity and sensitivity of the Sn/rGO/SPCE were evaluated for potential interferences for the analysis [40]. In a $130 \mu \mathrm{M}$ capsaicin standard solution, $2 \mathrm{mM}$ interfering species each in sodium acetate buffer solution ( $\mathrm{pH}$ 3.0) was added. \% Recovery of between 81\%-97\% was obtained, as shown in Fig. 7, proving that the interfering species did not have any influence on the capsaicin determination, and the $\mathrm{Sn} / \mathrm{rGO} / \mathrm{SPCE}$ was very selective to capsaicin. The prepared electrode was therefore suitable for quantitative determination of capsaicin. 


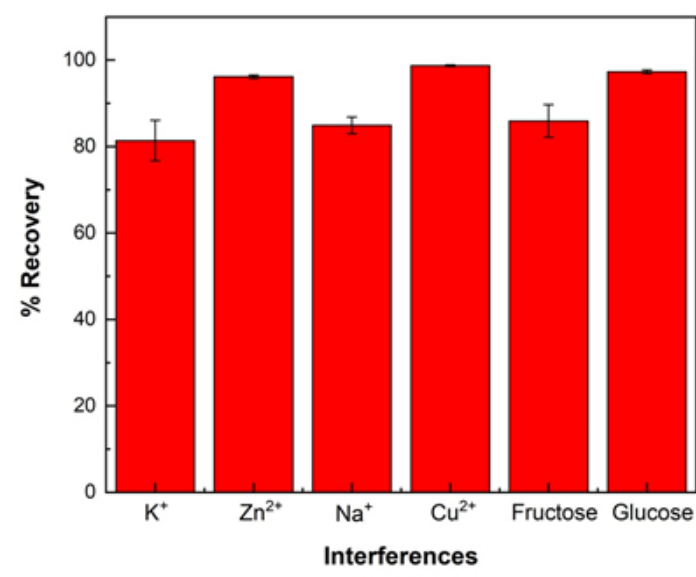

Fig. 7 Comparisons of $2 \mathrm{mM}$ interfering substances added into $130 \mu \mathrm{M}$ capsaicin in sodium acetate buffer solution (pH 3.0) under the optimized conditions of measurement using the $\mathrm{Sn} / \mathrm{rGO} / \mathrm{SPCE}$.

\section{Reproducibility and stability of Sn/rGO/SPCE}

To confirm the precision of the proposed electrode, the reproducibility and stability of $\mathrm{Sn} / \mathrm{rGO} / \mathrm{SPCE}$ were examined. Three electrodes prepared by the same procedure were used to determine $2 \mu \mathrm{M}$ and $22 \mu \mathrm{M}$ capsaicin in acetate buffer solution ( $\mathrm{pH} 3.0$ ). The relative standard deviation (RSD) for the anodic peak currents of $2 \mu \mathrm{M}$ and $22 \mu \mathrm{M}$ capsaicin were approximately $1.89 \%$ and $2.01 \%$, respectively, suggesting high reproducibility of the $\mathrm{Sn} / \mathrm{rGO} / \mathrm{SPCE}$. In addition, the stability of the electrode was evaluated by measuring the anodic peak current of $22 \mu \mathrm{M}$ capsaicin over a one week period. It was observed that the $\mathrm{Sn} / \mathrm{rGO}$ did not come off during measurement, assuring that the catalyst ink of $\mathrm{Sn} / \mathrm{rGO}$ was well-adhered to the surface of SPCE. The electrode was stored in a desiccator over the testing period. The anodic peak current of $22 \mu \mathrm{M}$ capsaicin in an acetate buffer solution (pH 3.0) decreased by $8.90 \%$ at the end of the week, indicating a high stability of the electrode. The intra-day measurement was carried out with three independent determinations of $10 \mu \mathrm{M}$ capsaicin in acetate buffer solution (pH 3.0), resulting in an excellent RSD of $2.02 \%$. Furthermore, the inter-day precision obtained by measuring anodic peak current for three consecutive days in $10 \mu \mathrm{M}$ capsaicin solution was satisfactorily achieved at $2.58 \%$ RSD.

\section{Electrochemical analysis of real samples}

Capsaicin in real samples, such as Karen chili, Bird's eye chili and chili sauces was also successfully determined by using the Sn/rGO/SPCE. Measurement results of spiked and non-spiked samples are shown in Table 2. The results for capsaicin determination in real samples of Karen chili, Bird's eye chili and chili sauces A and B were satisfactory with the recovery of $107.26,104.25,94.05$ and $94.10 \%$, respectively. Triplicate analyses of the samples were achieved with the reasonably good RSDs, $3.62 \%-7.66 \%$. The concentration in $\mu \mathrm{M}$ has been converted to ppm, and to Scoville units. A 1 ppm is approximately equal to 15 Scoville units. The results confirmed a potential application of the $\mathrm{Sn} / \mathrm{rGO} / \mathrm{SPCE}$ for determination of capsaicin in chili samples.

\section{CONCLUSION}

In the present work, the remarkable analytical sensitivity of the $\mathrm{Sn} / \mathrm{rGO} / \mathrm{SPCE}$, together with its simple preparation and economical cost, makes such the thin-film electrode having a great potential application as a capsaicin sensor for testing the heat in commercial food products. The oxidation of capsaicin produced derivatives that possibly adsorbed on the electrode surface, resulting in the loss of electrode performance during repeated experiment with the same electrode. The further advantage of the Sn/rGO/SPCE was hence its single-use, which eliminated the fouling problem of the electrode surface. In the extraction step of capsaicin from chili samples, some potential interfering species could be co-extracted; however, the dilution of high concentrated capsaicin extract with buffer was needed for detection at low concentration range. Any interfering species were, therefore, subsequently reduced during the measurement. These features clearly show that the proposed thin electrode successfully performed as the effective capsaicin electrochemical sensor for food and pharmaceuticals at a very lowcost.

\section{Appendix A. Supplementary data}

Supplementary data associated with this article can be found at http://dx.doi.org/10.2306/ scienceasia1513-1874.2020.076.

Acknowledgements: This work has been carried out under the auspices of the Center of Excellence for Innovation in Chemistry (PERCH-CIC), Commission on Higher Education, Ministry of Education. The Department of Chemistry, Faculty of Science, Kasetsart University is also acknowledged for instruments and facilities support. 
Table 2 Determination of capsaicin in real sample.

\begin{tabular}{lccrrrr}
\hline Sample & $\begin{array}{c}\text { Original value } \\
(\mu \mathrm{M})\end{array}$ & $\begin{array}{c}\text { Added } \\
(\mu \mathrm{M})\end{array}$ & $\begin{array}{c}\text { Found } \\
(\mu \mathrm{M})\end{array}$ & \% Recovery & $\begin{array}{c}\text { Capsaicin } \\
(\mu \mathrm{g} / \mathrm{ml})\end{array}$ & $\begin{array}{c}\text { Scoville } \\
\text { unit }\end{array}$ \\
\hline Karen chili & 4.68 & 4.94 & 9.96 & $107.26 \pm 4.13$ & 3.04 & 45.63 \\
Bird's eye chili $_{\text {Chili sauce A }}^{\mathrm{a}}$ & 4.70 & 5.16 & 10.06 & $104.25 \pm 4.04$ & 3.07 & 46.09 \\
Chili sauce B $^{\mathrm{a}}$ & 12.26 & 12.04 & 23.57 & $94.05 \pm 3.55$ & 7.20 & 107.98 \\
\hline
\end{tabular}

${ }^{\mathrm{a}} \mathrm{A}$ and $\mathrm{B}$ represented different manufacturers.

\section{REFERENCES}

1. Choi SH, Suh BS, Kozukue E, Kozukue N, Levin CE, Friedman M (2006) Analysis of the contents of pungent compounds in fresh Korean red peppers and in pepper-containing foods. J Agr Food Chem 54, 9024-9031.

2. Yardım Y, Şentürk Z (2013) Electrochemical evaluation and adsorptive stripping voltammetric determination of capsaicin or dihydrocapsaicin on a disposable pencil graphite electrode. Talanta 112, 11-19.

3. Higashiguchi F, Nakamura H, Hayashi H, Kometani $\mathrm{T}$ (2006) Purification and structure determination of glucosides of capsaicin and dihydrocapsaicin from various capsicum Fruits. J Agr Food Chem 54, 5948-5953.

4. Monforte-González M, Medina-Lara F, GutiérrezCarbajal G, Vázquez-Flota F (2007) capsaicinoid quantitation by in situ densitometry of thin layer chromatography plates. J Liq Chromatogr R T 30, 1697-1704.

5. Antonious GF, Berke T, Jarret RL (2009) Pungency in capsicum chinense: variation among countries of origin. J Environ Sci Heal B 44, 179-184.

6. Barbero GF, Liazid A, Palma M, Barroso CG (2008) Fast determination of capsaicinoids from peppers by high-performance liquid chromatography using a reversed phase monolithic column. Food Chem 107, 1276-1282.

7. Ohnuki K, Haramizu S, Oki K, Watanabe T, Yazawa S, Fushiki T (2001) Administration of capsiate, a non-pungent capsaicin analog, promotes energy metabolism and suppresses body fat accumulation in mice. Biosci Biotech Bioch 65, 2735-2740.

8. Sessa WC (2010) A new way to lower blood pressure: pass the chili peppers please! Cell Metab 12, 109-110.

9. Akerman S, Kaube H, Goadsby PJ (2003) Vanilloid type 1 receptors (VR1) on trigeminal sensory nerve fibres play a minor role in neurogenic dural vasodilatation, and are involved in capsaicin-induced dural dilation. Brit $J$ Pharmacol 140, 718-724.

10. Van der Schueren BJ, De Hoon JN, Vanmolkot FH, Van Hecken A, Depre M, Kane SA, Lepeleire ID, Sinclair SR (2007) Reproducibility of the capsaicin- induced dermal blood flow response as assessed by laser Doppler perfusion imaging. Brit $J$ Clin Pharmaco 64, 580-590.

11. Henderson DE, Slickman AM, Henderson SK (1999) Quantitative HPLC determination of the antioxidant activity of capsaicin on the formation of lipid hydroperoxides of linoleic acid: a comparative study against BHT and melatonin. J Agr Food Chem 47, 2563-2570.

12. Liu X, Lin T, Peng B, Wang X (2012) Antibacterial activity of capsaicin-coated wool fabric. Text Res $J \mathbf{8 2}$, 584-590.

13. Rosa A, Deiana M, Casu V, Paccagnini S, Appendino G, Ballero M, Dessi MA (2002) Antioxidant activity of capsinoids. J Agr Food Chem 50, 7396-7401.

14. Hawer WS, Ha J, Hwang J, Nam Y (1994) Effective separation and quantitative analysis of major heat principles in red pepper by capillary gas chromatography. Food Chem 49, 99-103.

15. Krajewska AM, Powers JJ (1987) Gas chromatography of methyl derivatives of naturally occurring capsaicinoids. J Chromatogr A 409, 223-233.

16. Reilly CA, Crouch DJ, Yost GS, Fatah AA (2001) Determination of capsaicin, dihydrocapsaicin, and nonivamide in self-defense weapons by liquid chromatography-mass spectrometry and liquid chromatography-tandem mass spectrometry. $J$ Chromatogr A 912, 259-267.

17. Charoenkitamorn K, Yakoh A, Jampasa S, Chaiyo S, Chailapakul O (2020) Electrochemical and optical biosensors for biological sensing applications. ScienceAsia 46, 245-253.

18. Hartati YW, Setiawati TA, Sofyatin T, Fitrilawati F, Anggraeni A, Gaffar S (2020) Electrochemical DNA biosensor for detection of pork (Sus scrof) using screen printed carbon-reduced graphene oxide electrode. ScienceAsia 46, 72-79.

19. Randviir EP, Metters JP, Stainton J, Banks CE (2013) Electrochemical impedance spectroscopy versus cyclic voltammetry for the electroanalytical sensing of capsaicin utilising screen printed carbon nanotube electrodes. Analyst 138, 2970-2981.

20. Kachoosangi RT, Wildgoose GG, Compton RG (2008) Carbon nanotube-based electrochemical sensors for quantifying the 'heat' of chilli peppers: the adsorptive stripping voltammetric determination of capsaicin. 
Analyst 133, 888-895.

21. Yardım Y (2011) Sensitive detection of capsaicin by adsorptive stripping voltammetry at a boron-doped diamond electrode in the presence of sodium dodecylsulfate. Electroanal 23, 2491-2497.

22. Manaia MAN, Diculescu VC, Gil EdS, Oliveira-Brett AM (2012) Guaicolic spices curcumin and capsaicin electrochemical oxidation behaviour at a glassy carbon electrode.J Electroanal Chem 682, 83-89.

23. Verma A, Jain R (2017) Ultrasensitive voltammetric quantification of antioxidant capsaicin at platform polypyrrole $/ \mathrm{Bi}_{2} \mathrm{O}_{3}$ /graphene oxide in surfactant stabilized media. $J$ Electrochem Soc 164, H908-H917.

24. Ping J, Wang Y, Fan K, Wu J, Ying Y (2011) Direct electrochemical reduction of graphene oxide on ionic liquid doped screen-printed electrode and its electrochemical biosensing application. Biosens Bioelectron 28, 204-209.

25. Wang Y, Huang B, Dai W, Ye J, Xu B (2016) Sensitive determination of capsaicin on $\mathrm{Ag} / \mathrm{Ag}_{2} \mathrm{O}$ nanoparticles/reduced graphene oxide modified screenprinted electrode. $J$ Electroanal Chem 776, 93-100.

26. Zhong F, Liu Z, Han Y, Guo Y (2019) Electrochemical sensor for sensitive determination of capsaicin using Pd decorated reduced graphene oxide. Electroanal 31, 1182-1188.

27. Ya Y, Mo L, Wang T, Fan Y, Liao J, Chen Z, et al (2012) Highly sensitive determination of capsaicin using a carbon paste electrode modified with aminofunctionalized mesoporous silica. Colloid Surface B 95, 90-95.

28. Baytak AK, Aslanoglu M (2017) Sensitive determination of capsaicin in pepper samples using a voltammetric platform based on carbon nanotubes and ruthenium nanoparticles. Food Chem 228, 152-157.

29. Fujishima M, Jin Q, Yamamoto H, Tada H, Nolan M (2012) Tin oxide-surface modified anatase titanium (IV) dioxide with enhanced UV-light photocatalytic activity. Phys Chem Chem Phys 14, 705-711.

30. Siriwatcharapiboon W, Kwon Y, Yang J, Chantry R, Li Z, Horswell SL, Koper MTM (2014) Promotion effects of $\mathrm{Sn}$ on the electrocatalytic reduction of nitrate at $\mathrm{Rh}$ nanoparticles. ChemElectroChem 1, 172-179.
31. Modarres MH, Lim JH-W, George C, De Volder M (2017) Evolution of reduced graphene oxide- $\mathrm{SnS}_{2}$ hybrid nanoparticle electrodes in Li-ion batteries. $J$ Phys Chem C 121, 13018-13024.

32. Rudnik E, Włoch G (2013) Studies on the electrodeposition of tin from acidic chloride-gluconate solutions. Appl Surf Sci 265, 839-849.

33. Ghann WE, Kang H, Uddin J, Chowdhury FA, Khondaker SI, Moniruzzaman M, et al (2019) Synthesis and characterization of reduced graphene oxide and their application in dye-sensitized solar cells. Chem Eng $J$ 3, ID 7

34. Tao P, Viswanath A, Schadler LS, Benicewicz BC, Siegel RW (2011) Preparation and optical properties of indium tin oxide/epoxy nanocomposites with polyglycidyl methacrylate grafted nanoparticles. ACS Appl Mater Inter 3, 3638-3645.

35. Al-Gaashani R, Najjar A, Zakaria Y, Mansour S, Atieh M (2019) XPS and structural studies of high quality graphene oxide and reduced graphene oxide prepared by different chemical oxidation methods. Ceram Int 45, 14439-14448.

36. Lu F, Ji X, Yang Y, Deng W, Banks CE (2013) Room temperature ionic liquid assisted well-dispersed coreshell tin nanoparticles through cathodic corrosion. RSC Adv 3, 18791-18793.

37. Wang X, Lian J, Wang Y (2014) The effect of Sn on platinum dispersion in Pt/graphene catalysts for the methanol oxidation reaction. Int $J$ Hydrogen Energ 39, 14288-14295.

38. Wang Y, Wang X, Wang Y, Li J (2015) Acid-treatmentassisted synthesis of Pt-Sn/graphene catalysts and their enhanced ethanol electro-catalytic activity. Int J Hydrogen Energ 40, 990-997.

39. Xue Z, Hu C, Rao H, Wang X, Zhou X, Liu X, et al (2015) A novel electrochemical sensor for capsaicin based on mesoporous cellular foams. Anal Methods 7, 1167-1174.

40. Temcheon P, Chienthavorn O, Siriwatcharapiboon W, Hasin P (2019) In situ formation of nitrogen doped mesoporous carbon via directly carbonizing polyaniline as an efficient electrocatalyst for determination of capsaicin. Micropor Mesopor Mat 278, 327-339. 


\section{Appendix A. Supplementary data}
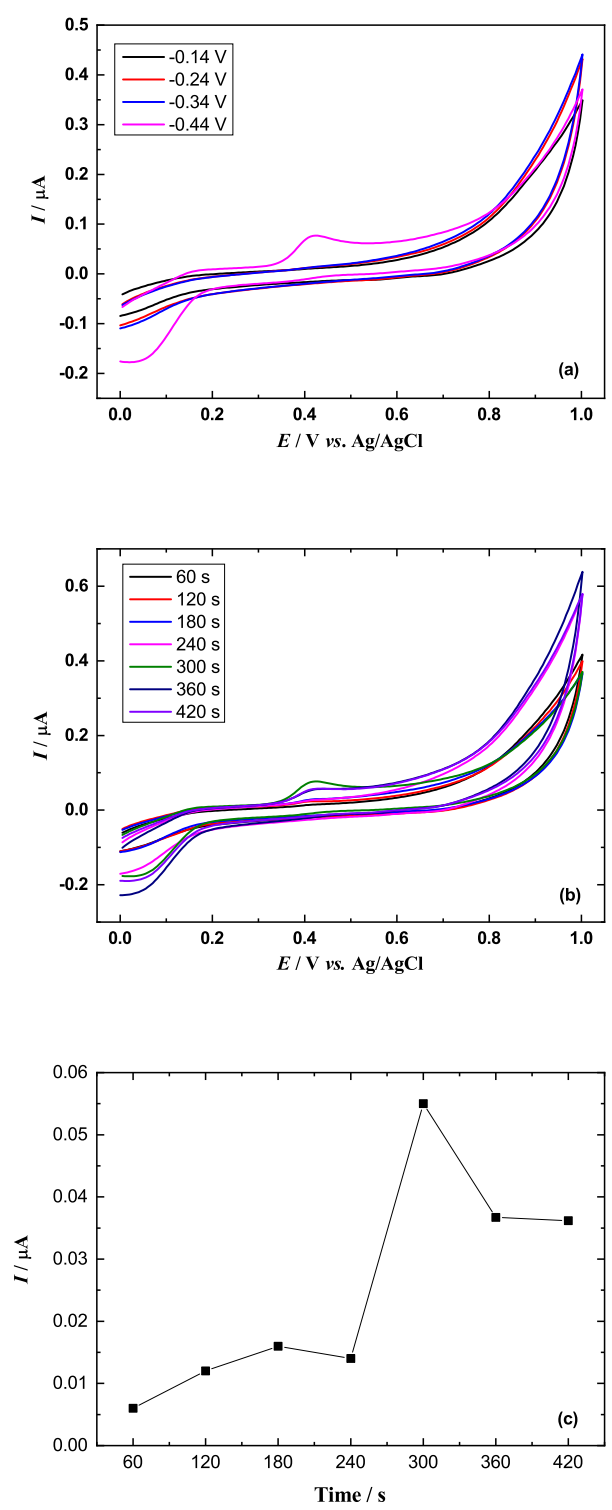

Fig. S1 (a) Cyclic voltammograms of different Sn electrodeposition potentials, (b) cyclic voltammograms of different Sn electrodeposition time, and (c) linear plots between time anodic peak current in $2 \mathrm{mM}$ capsaicin and sodium acetate buffer solution ( $\mathrm{pH} 3.0)$ at $50 \mathrm{mV} / \mathrm{s}$.

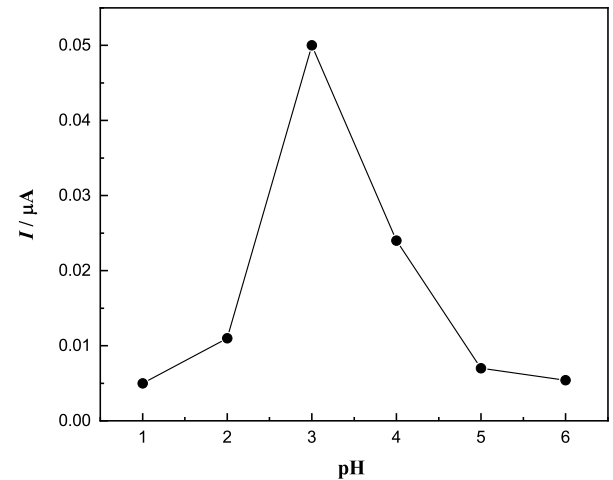

Fig. S2 Plot between anodic peak current of the $\mathrm{Sn} / \mathrm{rGO} / \mathrm{SPCE}$ in $2 \mathrm{mM}$ capsaicin and sodium acetate buffer solutions with different $\mathrm{pH}$ from 1.0 to 6.0 at $50 \mathrm{mV} / \mathrm{s}$. 